\title{
3D-spectroscopy of SBS star-forming galaxies
}

\author{
S. A. Hakopian ${ }^{1}$, S. N. Dodonov ${ }^{2}$, A. V. Moiseev ${ }^{2}$ and \\ A. A. Smirnova ${ }^{2}$ \\ ${ }^{1}$ Byurakan Astrophysical Observatory (BAO), Armenia \\ email:susannahakopian@yahoo.com \\ ${ }^{2}$ Russia Academy of Sciences (SAO RUS), Russia
}

\begin{abstract}
In the base of our studies of star-forming processes are the data provided by panoramic spectroscopy of galaxies composing our SBS subsample. Observations with multi-pupil spectrographs are organized in a way to obtain the spectral range centered in permitted hydrogen $\mathrm{H} \alpha$ Balmer line, by capturing at least the forbidden doublets of nitrogen [NII]6548,6583 and sulfur [SII] 6716,6731 . The results obtained are the spatial distributions across the targets of emission intensities and derived properties from line parameters such as radial velocities, which give us the possibility to explore gas kinematics and physical characteristics of HII regions.
\end{abstract}

Keywords. SBS, starforming galaxies

\section{Introduction}

The Second Byurakan Survey, SBS, has been carried with the Byurakan 1-m Schmidt type telescope after the conduction of the First Survey (FBS/Markarian's survey). The area of 65 SBS fields altogether $\left(1040 \mathrm{deg}^{2}\right)$ covered less than a 15 th part of FBS. With some changes in the methods of observation a limiting magnitude has been increased up to apparent magnitudes of $18^{m}-19.5^{m}(\mathrm{pg})$, which is 2-mag fainter in average than that of FBS. While the only criterion in FBS was to have a strong UV continuum, in the SBS the presence of line emission was added. The selected extragalactic objects were divided (preliminary) into two samples, QSO and galaxies, in accordance to their star-like or diffuse appearance, correspondingly. Our current investigations are related to the sample of galaxies of the Second survey.

\section{SEVEN SBS FIELDS and the BASIC SAMPLE}

Because of a complicated observational program with several steps to be performed, the quality of the resulting field samples fluctuates, this being dependent on the number of plates obtained and on the seeing conditions. Targets from a total of seven fields were selected (with 500 galaxies in total) conforming our basic sample for forthcoming studies, including the follow-up spectroscopy that should provide a classification based on nuclear activity. The galaxies showing star-forming activity are designated as SfG (Star-forming Galaxy). To avoid the heterogeneity of classifications found in the literature, two simple subclasses, SfGcontinual with EW H $\alpha<100$ and SfGnebular (the most of which are Blue Compact Galaxies), are currently used. Our aim is to develop a detailed classification scheme by using our SfG subsample of galaxies. It composed of about $65 \%$ of SfG in the basic list, namely 324 galaxies. So there is no doubt on its representativeness in the variety of objects in different stages of activity and morphological structure, including line emission, stellar haloes and environment. Detailed investigations of individual objects to fill in the space of parameters for further statistics is currently ongoing. 